\title{
GENERATING MODULES EFFICIENTLY OVER NONCOMMUTATIVE NOETHERIAN RINGS
}

\author{
S. C. COUTINHO
}

\begin{abstract}
The Forster-Swan Theorem gives an upper bound on the number of generators of a module over a commutative ring in terms of local data. Stafford showed that this theorem could be generalized to arbitrary right and left noetherian rings. In this paper a similar result is proved for right noetherian rings with finite Krull dimension. A new dimension function-the basic dimension-is the main tool used in the proof of this result.
\end{abstract}

\section{INTRODUCTION}

The proof of many interesting results in commutative algebra and algebraic $K$-theory are applications of local-global principles. These are results of the following type: if a property is true for all the localisations of an $R$-module $M$ at each prime ideal of $R$, then it is true for $M$ itself. For example, a principle of this kind lies at the heart of Quillen's solution of Serre's conjecture [8]. A further example of a local-global principle is the Forster-Swan Theorem. Suppose that $M$ is a finitely generated right module over a right noetherian ring $R$. Let us denote the minimal number of generators of $M$ by $g_{R}(M)$. For a commutative ring $R$, the Forster-Swan Theorem gives an upper bound for $g_{R}(M)$ in terms of local data; more precisely

$$
g_{R}(M) \leq \sup \left\{g_{R_{P}}\left(M_{P}\right)+\operatorname{Kdim}(R / P): P \text { a } J \text {-prime ideal of } R\right\} .
$$

For a proof of this theorem and some of its applications to commutative algebra and algebraic geometry, see [8].

In this paper we are interested in a generalisation of the above inequality to noncommutative noetherian rings. However, before this generalisation is achieved, certain preliminary difficulties-the choice of an appropriate dimension function and the lack of localisation-have to be overcome. For the dimension function the natural choice is the Krull dimension as defined by Gabriel and Rentschler (see [5] for definition and properties). Turning now to consider the localisation problem, if $R$ is commutative, an application of Nakayama's Lemma shows that for a prime ideal $P$ of $R$, one has

$$
g_{R_{P}}\left(M_{P}\right)=g_{(R / P)_{P}}\left(M / M P \otimes_{R / P}(R / P)_{P}\right) .
$$

Received by the editors February 10, 1989.

1980 Mathematics Subject Classification (1985 Revision). Primary 16A33, 19A15, 19A13. 
Note that $(R / P)_{P}$ is the quotient ring of the domain $R / P$. This latter construction is available in the noncommutative case since, by Goldie's Theorem, a prime right noetherian ring admits a simple artinian quotient ring. This suggests the following definition. Let $M$ be a finitely generated right module over a right noetherian ring $R$ and let $P$ be a prime ideal of $R$. The 'local number of generators of $M$ at $P^{\prime}$, denoted $g(M, P)$, is given by

$$
g(M, P)=g_{Q(R / P)}\left(M / M P \otimes_{R / P} Q(R / P)\right)
$$

where $Q(R / P)$ is the Goldie quotient ring of the prime ring $R / P$.

The definition of $g(M, P)$ given above is that of Warfield [14], who also showed that this definition can be used to establish a version of the Forster-Swan Theorem for FBN rings. Stafford [11] generalised Warfield's work considerably and proved the following result:

Theorem (Stafford [11, Theorem 3.1]). Let $M$ be a finitely generated right module over a right and left noetherian ring $R$. Then

$$
g_{R}(M) \leq \sup \{g(M, P)+\mathrm{K} \operatorname{dim}(R / P): P \text { a } J \text {-prime ideal of } R\} .
$$

The proof of this theorem in [11] is extremely technical. However, Stafford showed that many of these technical difficulties can be avoided if it is assumed that the ring $R$ satisfies the weak ideal invariance condition, see [12]. Unfortunately, right and left noetherian rings which are not weakly ideal invariant do exist, see [13].

In this paper we take a different point of view. Instead of imposing additional conditions on the ring, we work with a different dimension function, for which all right noetherian rings are weakly ideal invariant. This is the basic dimension, as defined and studied in $\S \S 1$ and 2 . Using this dimension we are able to prove a version of the Forster-Swan Theorem for right noetherian rings with finite right Krull dimension (Theorem 5.4). In $\S 3$ we present again the terminology adopted in [11], necessary to state the theorem in its full generality. The proof of our version of the Forster-Swan Theorem itself is given in $\S 5$.

Several applications of the Forster-Swan Theorem to the study of noncommutative rings can be found in [11]. The reader should take particular notice of $[11, \S 5]$, where generalisations of Serre's Theorem and Bass's Cancellation Theorem are to be found. The applications contained in [11] are there stated for right and left noetherian rings. However, using Theorem 5.4 the same results can be established, with essentially the same proofs, for right noetherian rings of finite Krull dimension, see [1]. Recently, Dean and Stafford [3] used the Forster-Swan Theorem to show that a certain right and left noetherian ring cannot be embedded in an artinian ring. In a subsequent paper [2], we show how the basic dimension can be used to develop a theory of basic elements for noncommutative noetherian rings. 
Finally we would like to mention that some of the results presented in this paper, although in less general form, have appeared in the books [9] and [10].

\section{BASIC DIMENSION}

It is a well-known fact that, over a noncommutative ring, a torsion module can be faithful. In this section we study a variant of the Krull dimension which measures how close such a module is to having a nonzero annihilator.

Let $n \geq-1$ be an integer and $M$ a noetherian module. An $n$-basic composition series of $M$ is a series of submodules

$$
M=M_{s} \supset M_{s-1} \supset \cdots \supset M_{0}=0
$$

such that if $E=\left\{i: 1 \leq i \leq s\right.$ and $\left.r-\operatorname{ann}_{R}\left(M_{i} / M_{i-1}\right)=0\right\}$ then:

(a) $M_{i} / M_{i-1}$ is critical for every $i \in E$ and,

(b) $n=\max \left\{\operatorname{Kdim}\left(M_{i} / M_{i-1}\right): i \in E\right\}$.

Note that $n=-1$ is equivalent to $E$ being empty. That $M$ has an $n$-basic composition series for some $n$ follows from the fact that a noetherian module always has a critical composition series.

Let $M$ be a noetherian right module over a ring $R$. The basic dimension of $M$ over $R$, denoted by $\operatorname{Bdim}_{R}(M)$, is the least $n \geq-1$ such that $M$ admits an $n$-basic composition series. Our first result outlines some of the properties of the basic dimension. The proof is an immediate consequence of the analogous result for Krull dimension and will be omitted.

Proposition 1.1. Let $M$ be a noetherian right module over a ring $R$.

(a) If $N$ is a submodule of $M$ then

$$
\operatorname{Bdim}_{R}(M)=\max \left\{\operatorname{Bdim}_{R}(N), \operatorname{Bdim}_{R}(M / N)\right\} .
$$

(b) If $r$-ann ${ }_{R}(M) \neq 0$ then $\operatorname{Bdim}_{R}(M)=-1$; and if $R$ is prime, the converse is true.

(c) If $R$ has right Krull dimension and $M$ is finitely generated over $R$, then $\operatorname{Bdim}_{R}(M) \leq \operatorname{Kdim}_{R}(M) \leq \operatorname{Kdim}(R)$.

Recall that a ring $R$ with right Krull dimension is said to be right weakly ideal invariant if, given any two-sided ideal $T$ and finitely generated right $R$-module $M$ such that $\operatorname{Kdim}(M)<\operatorname{Kdim}(R / T)$, then $\operatorname{Kdim}\left(M \otimes_{R} T\right)<\operatorname{Kdim}(R / T)$. As was indicated in the Introduction, noetherian rings which are not weakly ideal invariant do exist. However, if the basic dimension is used instead of Krull dimension, then all noetherian rings are weakly ideal invariant. In fact, as the next proposition shows, they satisfy an even stronger condition.

Proposition 1.2. Let $M$ be a finitely generated right module over a right noetherian ring $R$. If $T$ is a two-sided ideal of $R$ then $\operatorname{Bdim}_{R}(M)=\operatorname{Bdim}_{R}\left(M \otimes_{R} T\right)$. Proof. Without loss of generality suppose that $M$ is cyclic and let $M \simeq R / I$, for some right ideal $I$ of $R$. Since $M \otimes T \simeq T / I T$, it is sufficient to show that $\operatorname{Bdim}_{R}(T / I T)=\operatorname{Bdim}_{R}(R / I)$. By Proposition 1.1(a) we have

$$
\operatorname{Bdim}_{R}(R / I T)=\max \left\{\operatorname{Bdim}_{R}(R / T), \operatorname{Bdim}_{R}(T / I T)\right\}
$$


and

$$
\operatorname{Bdim}_{R}(R / I T)=\max \left\{\operatorname{Bdim}_{R}(R / I), \operatorname{Bdim}_{R}(I / I T)\right\} .
$$

Since $\operatorname{Bdim}_{R}(R / T)=\operatorname{Bdim}_{R}(I / I T)=-1$ by Proposition 1.1(b), it follows that $\operatorname{Bdim}_{R}(T / I T)=\operatorname{Bdim}_{R}(R / I T)=\operatorname{Bdim}_{R}(R / I)$, as desired.

Proposition 1.3. Let $M$ be a finitely generated right module over a prime right noetherian ring $R$.

(a) $\operatorname{Bdim}_{R}(M)=\mathrm{Kdim}(R)$ if and only if $M$ is not a torsion module.

(b) If $R$ is commutative or FBN then $\operatorname{Bdim}_{R}(M)$ equals -1 if $M$ is torsion, and equals $\mathrm{Kdim}(R)$ otherwise.

Proof. This is a consequence of [7, Proposition 1.4], according to which $M$ is torsion if and only if $\operatorname{Kdim}(M)<\operatorname{Kdim}(R)$. Thus if $M$ is torsion, $\operatorname{Bdim}_{R}(M)$ $\leq \mathrm{Kdim}(M)<\mathrm{Kdim}(R)$. On the other hand, if $\operatorname{Bdim}_{R}(M)<\mathrm{Kdim}(R)$, then for each factor $M_{i} / M_{i-1}$ in a basic composition series of $M$, either

$$
r-\operatorname{ann}_{R}\left(M_{i} / M_{i-1}\right) \neq 0
$$

or

$$
\operatorname{Kdim}\left(M_{i} / M_{i-1}\right) \leq \operatorname{Bdim}_{R}(M)<\operatorname{Kdim}(R) .
$$

In either case $M_{i} / M_{i-1}$ is torsion, hence so is $M$. Finally (b) holds because if $R$ is FBN then $M$ is torsion if and only if $r$-ann ${ }_{R}(M) \neq 0$.

Let $R$ be a right noetherian ring and $M$ a finitely generated right $R$-module. If $P$ is a prime ideal of $R$, set $\operatorname{Bdim}(M, P)=\operatorname{Bdim}_{R / P}(M / M P)$. If $X \subseteq$ $\operatorname{Spec}(R)$ then we will extend the previous notation and write $\operatorname{Bdim}(M, X)=$ $\sup \{\operatorname{Bdim}(M, P): P \in X\}$. It is $\operatorname{Bdim}(M, P)$ that we will use to formulate and prove the results of later sections. The next corollaries follow immediately from Propositions 1.1 and 1.3.

Corollary 1.4. Let $M$ be a finitely generated right module over a right noetherian ring $R$. If $P$ is a prime ideal of $R$ then

(a) $-1 \leq \operatorname{Bdim}(M, P) \leq \mathrm{K} \operatorname{dim}(M / M P) \leq \mathrm{Kdim}(R / P)$.

(b) $\operatorname{Bdim}(M, P)=-1$ if and only if $r$-ann ${ }_{R}(M / M P) \neq P$.

(c) $\operatorname{Bdim}(M, P)=\mathrm{Kdim}(R / P)$ if and only if $M / M P$ is not torsion over $R / P$.

Corollary 1.5. Let $M$ and $R$ be as in Corollary 1.4. If $X \subseteq \operatorname{Spec}(R)$ and $N$ is a submodule of $M$ then

(a) $\operatorname{Bdim}(M, X) \leq \operatorname{Kdim}(R)$.

(b) $\operatorname{Bdim}(M / N, X) \leq \operatorname{Bdim}(M, X) \leq \max \{\operatorname{Bdim}(N, X), \operatorname{Bdim}(M / N, X)\}$.

\section{Patch-Closed Sets}

Throughout this section let $R$ be a right noetherian ring and $M$ a finitely generated right $R$-module. If $I \subseteq R$, set $V(I)=\{P \in \operatorname{Spec}(R): I \subseteq P\}$ and $W(I)=\{P \in \operatorname{Spec}(R): I \nsubseteq P\}$. Recall that the patch topology on $\operatorname{Spec}(R)$ is the topology which has a subbasis of closed sets consisting of $V(I)$ and $W(I)$ 
for each two-sided ideal $I$ of $R$. There is a very neat description of the patchclosed sets which will be given in the next proposition.

Proposition 2.1. A set $X \subseteq \operatorname{Spec}(R)$ is patch-closed if and only if any prime in $\operatorname{Spec}(R)$ which is an intersection of prime ideals in $X$ belongs to $X$.

Proof. Clearly $V(I)$ and $W(I)$ satisfy this condition. We prove the converse. Suppose that $X \subseteq \operatorname{Spec}(R)$ satisfies this condition and let $X^{\prime}$ be its complement in $\operatorname{Spec}(R)$. For each $Q \in X^{\prime}$, let $I_{Q}=\bigcap\{P \in X: Q \subseteq P\}$. By definition $Q \subset I_{Q}$ and so $Q \in V(Q) \cap W\left(I_{Q}\right)$. Since $V(Q) \cap W\left(I_{Q}\right) \subset X^{\prime}$ it follows that $X^{\prime}$ is the union of such subsets, each of which is open in the patch topology. Hence $X^{\prime}$ is open, and $X$ closed, in the patch topology.

If $X$ is patch-closed and $t$ an integer, write $X_{t}=\{P \in X: r-\operatorname{Kdim}(R / P) \geq$ $t\}$. Note that $\operatorname{Spec}(R)$ is always patch-closed, and so too is the $J$-Spec of a commutative ring. The next proposition indicates that in the noncommutative case $J$-Spec should include the primitive ideals of the ring. Recall that an ideal $P$ of $R$ is right primitive if there exists a faithful simple right $R / P$-module.

Proposition 2.2. If $\operatorname{Bdim}(M, P)=-1$ for every right primitive ideal $P$ of $R$, then $M=0$.

Proof. Suppose that $M \neq 0$ and choose a two-sided ideal $I$ of $R$ maximal with respect to having $M \neq M I$. Let $N$ be a maximal submodule of $M$ with $M I \subset N$ and let $P=r$-ann ${ }_{R}(M / N)$. Then $P$ is right primitive and, since $M P \subset N$, and $I \varsubsetneqq P$ we have $r$-ann $(M / M P)=P$. Therefore, by Proposition 1.4(b), $\operatorname{Bdim}(M, P) \neq-1$, a contradiction.

Now let $J-\operatorname{Spec}(R)$ be the set of all prime ideals of $R$ which are intersections of right primitive ideals. In other words, $J-\operatorname{Spec}(R)$ is the smallest patch-closed subset of $\operatorname{Spec}(R)$ which contains all the right primitive ideals of $R$. Notice that $J-\operatorname{Spec}(R)$ contains also the left primitive ideals of $R$; in fact it is the smallest patch-closed set which contains these ideals. See [6, pp. 192-196] for details. The elements of $\operatorname{Spec}(R)$ will be called $J$-primes.

The importance of patch-closed sets lies in the following property: if $X \subseteq$ $\operatorname{Spec}(R)$ is patch-closed and $J-\operatorname{Spec}(R) \subseteq X$ then $\operatorname{Bdim}(M, X)=\operatorname{Bdim}(M, P)$ holds for only finitely many primes $P \in X$. The crucial step in the proof of this and other finiteness properties of the basic dimension is isolated in the next theorem. First a technical lemma.

Lemma 2.3. Let $L \subset N$ be submodules of $M$ with $N / L$ critical. If $I$ is a two-sided ideal of $R$ and $I \not \subset r$-ann $(N / L)$, then $\operatorname{Kdim}(N+M I / L+M I)<$ $\operatorname{Kdim}(N / L)$.

Proof. Since $I \not \subset r$-ann $(N / L)$, then $N I \not \subset L$. Hence $\operatorname{Kdim}(N / N I+L)<$ $\operatorname{Kdim}(N / L)$ by the criticality of $N / L$. Thus $\operatorname{Kdim}(N / N \cap(M I+L)) \leq$ $\mathrm{K} \operatorname{dim}(N / N I+L)<\operatorname{Kdim}(N / L)$, as required.

Theorem 2.4. Let $R$ be a prime right noetherian ring and $M$ be a finitely generated right $R$-module. Suppose that $\operatorname{Bdim}(M, 0) \leq s$, for some integer 
$s \geq 0$, and let $X$ be an infinite set of nonzero prime ideals of $R$ such that $\operatorname{Bdim}(M, P) \geq s$ for each $P \in X$. Then

$$
\bigcap\{P: P \in X\} \neq 0 \text {. }
$$

Proof. If $\operatorname{Bdim}(M, 0)=-1$ then $I=r-\operatorname{ann}_{R}(M) \neq 0$ and since $\operatorname{Bdim}(M, P)$ $\geq 0$ for $P \in X$, it follows that $I \subset \bigcap\{P: P \in X\}$ as required. Thus we may assume that $\operatorname{Bdim}(M, 0)=n \neq-1$. Let

$$
M=M_{v} \supset M_{v-1} \supset \cdots \supset M_{0}=0
$$

be an $n$-basic composition series of $M$. Let $J_{i}=r$-ann ${ }_{R}\left(M_{i} / M_{i-1}\right)$, and $J=\bigcap\left\{J_{i}: J_{i} \neq 0\right.$ and $\left.1 \leq i \leq v\right\}$. We claim that $J \subset \bigcap\{P: P \in X\}$. Since, clearly, $J \neq 0$, this is enough to show that the conclusion of the theorem holds. We proceed to prove the claim.

Let $P \in X$. One of the hypothesis of the theorem is that $\operatorname{Bdim}(M, P) \geq s$. Applying 1.5(b) to the series (2.5) we conclude that, for some $i$,

$$
\operatorname{Bdim}\left(M_{i}+M P / M_{i-1}+M P, P\right) \geq s \text {. }
$$

However, if $J_{i} \not \subset P$, then $M_{i}+M P / M_{i-1}+M P$ is unfaithful as an $R / P$ module so has basic dimension -1 . Also, if $J_{i}=0$, then using Lemma 2.3 one shows that $\operatorname{Bdim}\left(M_{i}+M P / M_{i-1}+M P, P\right)<s$. Hence, for some $i$, $0 \neq J_{i} \subset P$, and therefore $J \subset P$. The claim now follows immediately.

Corollary 2.6. Let $X$ be a patch-closed subset of $\operatorname{Spec}(R)$ and let $t \geq 0$ be an integer. Then there are only finitely many prime ideals $P \in X_{t}-X_{t+1}$ with

$$
\operatorname{Bdim}(M, P) \geq \max \left\{0, \operatorname{Bdim}\left(M, X_{t+1}\right)\right\} .
$$

Proof. Suppose that the result is false. Then the set

$$
Y=\left\{P \in X_{t}-X_{t+1}: \operatorname{Bdim}(M, P) \geq \max \left\{0, \operatorname{Bdim}\left(M, X_{t+1}\right)\right\}\right\}
$$

is infinite. Let $Q$ be an ideal maximal among the intersections of infinitely many elements of $Y$. Then $Q$ is prime and $\operatorname{Kdim}(R / Q)>t$. Further, since $X$ is patch-closed, $Q \in X_{t}$. Let $Y^{\prime}=\{P \in Y: Q \subset P\}$; by the choice of $Q, Y^{\prime}$ is infinite. Passing to the quotient ring $R / Q$, we can assume that $Q=0$. Applying Theorem 2.4 , we get $\bigcap\left\{P: P \in Y^{\prime}\right\} \neq 0$, which contradicts the maximality of $Q$.

Corollary 2.7. Let $\operatorname{Kdim}(R)<\infty$. If $M \neq 0$ then

$$
\operatorname{Bdim}(M, P)=\operatorname{Bdim}(M, J-\operatorname{Spec}(R))
$$

holds for only finitely many $J$-primes $P$.

Proof. This is a simple induction using Corollary 2.6. Note that since $M \neq 0$, $\operatorname{Bdim}(M, J-\operatorname{Spec}(R)) \geq 0$ by Proposition 1.1.

\section{HOMOMORPHIC IMAGES OF PROJECTIVE MODULES}

In this section we review the results of $[11, \S 2]$ concerning homomorphisms from a projective module to a finitely generated module. Let $M$ be a finitely 
generated right module over a right noetherian ring $R$ and $F$ a finitely generated projective right $R$-module. First we recall some standard notation. Let $P$ be a prime ideal of $R$ and $Q(R / P)$ the quotient ring of $R / P$. Then

$$
\rho(M, P)=\text { length }_{Q(R / P)}\left(M / M P \otimes_{R / P} Q(R / P)\right)
$$

is called the reduced rank of $M$ at $P$. If $R$ is prime, we will write $\rho_{R}(M)$ instead of $\rho(M, 0)$. Now set

$$
\begin{aligned}
\hat{g}^{F}(M, P) & =0 \text { if } \rho(M, P)=0 \\
& =\infty \quad \text { if } \rho(M, P) \neq 0 \text { but } \rho(F, P)=0 \\
& =\rho(M, P) / \rho(F, P) \quad \text { otherwise. }
\end{aligned}
$$

Define $g^{F}(M, P)$ to be the smallest integer greater or equal than $\widehat{g}^{F}(M, P)$. Notice that if $F=R$ then $g^{R}(M, P)=g(M, P)$, in the notation of the introduction. Similarly $\widehat{g}^{R}(M, P)=\widehat{g}(M, P)=\rho(M, P) / \rho(R, P)$ is the normalised rank of $M$ at $P$.

There is another way of defining $g^{F}(M, P)$ which is sometimes useful. Set $g^{F}(M)$ to be the smallest integer $n$ such that $F^{n}$ maps onto $M$, or $g^{F}(M)=$ $\infty$ if no such integer exists. Then it is readily checked that

$$
g^{F}(M, P)=g^{G}\left(M / M P \otimes_{R / P} Q(R / P)\right)
$$

where $G=F / F P \otimes_{R / P} Q(R / P)$. These definitions appeared originally in Warfield's paper [14], but we follow the notation of [11]. The following properties of $\widehat{g}^{F}(M, P)$ are easily checked: if $N$ is a submodule of $M$, then

$$
\widehat{g}^{F}(M, P) \leq \widehat{g}^{F}(N, P)+\widehat{g}^{F}(M / N, P) \text { and }
$$

if $R$ is prime then $\widehat{g}^{F}(M, 0)=\widehat{g}^{F}(N, 0)+\widehat{g}^{F}(M / N, 0)$.

Inspired by the Forster-Swan Theorem we would like to show that $g^{F}(M)$ can be bound above by means of $\left\{g^{F}(M, P): P \in \operatorname{Spec}(R)\right\}$. However, the following example from [11] shows that this is not always possible.

Example 3.3. Let $A$ be a simple, noetherian, nonartinian ring and $k$ a field. Set $R=A \oplus k, F=0 \oplus k$ and let $M$ be a simple $A$-module on which $k$ acts trivially. Then $g(M, 0)=0$ for all prime ideals $P$ of $R$, yet $g^{F}(M)=\infty$.

Thus it will be necessary to impose some condition on the projective module $F$ to guarantee that $M$ is 'finitely generated' in the sense that there exist $\theta_{i} \in$ $\operatorname{Hom}(F, M)$ for which $M=\sum_{1}^{n} \theta_{i}(F)$. This is done by the following definition from [11]. A finitely generated projective $R$-module $F$ is said to cover $M$ if every simple image of $M$ is also a simple image of $F$. Notice that if $F$ covers $M$ then it covers every homomorphic image of $M$.

Proposition 3.4 [11, Lemma 2.3]. $g^{F}(M)<\infty$ if and only if $F$ covers $M$.

An immediate consequence of Proposition 3.4 is that if $F$ covers $M$ then $g^{F}(M, P)$ is always finite for all prime ideals $P$ of $R$. Note that a free 
module covers every finitely generated $R$-module. Other situations for which this condition is automatic can be found in [11, Lemma 2.1].

We finish this section with some more notation. The first is a generalisation of the stable rank of a module. We define $s^{F}(M)$ by saying that $s^{F}(M) \leq s$ if given any integer $r>s$ and homomorphisms $\theta_{1}, \ldots, \theta_{r} \in \operatorname{Hom}(F, M)$ for which $M=\sum_{1}^{r} \theta_{i}(F)$, then there exist $\delta_{i} \in \operatorname{End}(F)$ such that

$$
M=\sum_{1}^{r-1}\left(\theta_{i}+\theta_{r} \delta_{i}\right)(F) .
$$

Next we define the function which will give the upper bound of the ForsterSwan inequality. Let $P$ be a prime ideal of $R$, set

$$
\begin{aligned}
b^{F}(M, P) & =g^{F}(M, P)+\mathrm{Kdim}(R / P) & & \text { if } g^{F}(M, P) \neq 0, \\
& =\operatorname{Bdim}(M, P)+1 & & \text { otherwise. }
\end{aligned}
$$

This differs from Stafford's notation in [11]; he has $b^{F}(M, P)=0$ if $g^{F}(M, P)$ $=0$. Note that if $g^{F}(M, P) \neq 0$ then $g^{F}(M, P)+\operatorname{Kdim}(R / P) \geq \operatorname{Bdim}(M, P)$ +1 and that equality holds if $g^{F}(M, P)=1$. For a subset $X$ of $\operatorname{Spec}(R)$, let

$$
b^{F}(M, X)=\sup \left\{b^{F}(M, P): P \in X\right\} .
$$

We can now write the inequality we are aiming at as

$$
s^{F}(M) \leq b^{F}(M, J-\operatorname{Spec}(R)) .
$$

\section{THE PRIME RING CASE}

Throughout this section let $M$ be a finitely generated right module over a prime right noetherian ring $R$ and let $F$ be a finitely generated projective right $R$-module. We begin with a generalisation of the well-known fact that if $J$ is a right ideal of $R$ then there exists $x \in J$ with uniform dimension $(x R)=$ uniform dimension $(J)$.

Proposition 4.1. Let $I$ be a nonzero two-sided ideal of $R$ and let $\alpha, \beta \in$ $\operatorname{Hom}(F, M)$. Then there exists $\delta: F \rightarrow F I$ such that

$$
\rho_{R}(M /(\alpha+\beta \delta)(F))=\max \left\{\rho_{R}(M / \alpha(F)+\beta(F)), \rho_{R}(M)-\rho_{R}(F)\right\} .
$$

Proof. Clearly the right-hand side is less or equal than the left-hand side. We prove the other inequality. Note that, since we are only interested in reduced ranks, the module $M$ can be assumed to be torsion free. Now choose $\delta: F \rightarrow$ $F I$ such that $\rho_{R}((\alpha+\beta \delta)(F))$ is as large as possible. Without loss of generality, replace $\alpha$ by $\alpha+\beta \delta$. If $\operatorname{Ker}(\alpha)=0$, then $\alpha(F) \simeq F$ and

$$
\rho_{R}(M / \alpha(F)+\beta(F)) \leq \rho_{R}(M)-\rho_{R}(F) .
$$

Thus $\delta=0$ will be satisfactory.

Now suppose that $\operatorname{Ker}(\alpha) \neq 0$ and that $\rho_{R}(\alpha(F)+\beta(F) / \alpha(F)) \neq 0$. In particular $\alpha(F)$ is not essential in $\alpha(F)+\beta(F)$. Choose a uniform submodule 
$D \subset \beta(F)$ such that $D \cap \alpha(F)=0$. Let $k$ be a nonzero element of $\operatorname{Ker}(\alpha)$. Since $F$ is projective, there is a map $\eta: F \rightarrow R$ with $\eta(k) \neq 0$. Hence $D I \eta(k) R \neq 0$, since $R$ is prime. Therefore $\beta(g) i \eta(k) \neq 0$ for some $g \in F$ with $\beta(g) \in D$ and $i \in I$. Define $\delta: F \rightarrow F I$ by $f \rightarrow \operatorname{gi\eta }(f)$. Then $0 \neq \beta(g) i \eta(k) \in D \cap(\alpha+\beta \delta)(F)$. Since $D$ is uniform $\alpha(F)+D /(\alpha+\beta \delta)(F)$ is torsion. Thus $\rho_{R}((\alpha+\beta \delta)(F))=\rho_{R}(\alpha(F))+1$, which contradicts the choice of $\alpha$. Hence $\rho_{R}(\alpha(F)+\beta(F) / \alpha(F))=0$, which completes the proof.

An easy induction argument yields the following result.

Corollary 4.2. Let $I$ be a nonzero two-sided ideal of $R$. If $M=\sum_{1}^{s} \theta_{i}(F)$ for $\theta_{i} \in \operatorname{Hom}(F, M)$ then there exist $\delta_{i} \in \operatorname{Hom}(F, F I)$ such that

$$
\rho\left(M /\left(\theta_{1}+\sum_{2}^{s} \theta_{i} \delta_{i}\right)(F)\right)=\max \left\{0, \rho_{R}(M)-\rho_{R}(F)\right\} .
$$

The next proposition is the analogue for $\operatorname{Bdim}_{R}(M)$ of Proposition 4.1.

Proposition 4.3. Let $I$ be a nonzero two-sided ideal of $R$. Suppose that $M$ is torsion and that $\alpha, \beta \in \operatorname{Hom}(F, M)$ satisfy $\operatorname{Bdim}_{R}(M / \alpha(F)+\beta(F))<$ $\operatorname{Bdim}_{R}(M)$. Then there exists $\delta: F \rightarrow F I$ such that

$$
\operatorname{Bdim}_{R}(M /(\alpha+\beta \delta)(F))<\operatorname{Bdim}_{R}(M) .
$$

Proof. By Proposition 1.1(a), $\operatorname{Bdim}_{R}(\beta(F I)) \leq \operatorname{Bdim}_{R}(M)$. Let

$$
0=N_{0} \subseteq N_{1} \subseteq \cdots \subseteq N_{s}=\beta(F I)
$$

be a basic composition series of $\beta(F I)$ and consider the inductive statement:

$A(i)$ : There exists $\delta \in \operatorname{Hom}(F, F I)$ with $\operatorname{Bdim}_{R}\left(M / N_{i}+(\alpha+\beta \delta)(F)\right)<$ $\operatorname{Bdim}_{R}(M)$.

Note first that

$$
\operatorname{Bdim}_{R}(\alpha(F)+\beta(F) / \alpha(F)+\beta(F I))=-1,
$$

since $I \subset r$-ann $(\alpha(F)+\beta(F) / \alpha(F)+\beta(F I))$. Therefore by Proposition 1.1(b)

$$
\operatorname{Bdim}_{R}(M / \alpha(F)+\beta(F I))=\operatorname{Bdim}_{R}(M / \alpha(F)+\beta(F))<\operatorname{Bdim}_{R}(M)
$$

and so $A(s)$ holds with $\delta=0$. Note also that $A(0)$ is the result at which we aim.

Suppose now that $A(i)$ is true for some $0<i \leq s$. We aim to show that $A(i-1)$ is also true. Let $\delta \in \operatorname{Hom}(F, F I)$ be as in $A(i)$. Without loss of generality we can replace $\alpha$ by $\alpha+\beta \delta$.

Let $L_{i}=r-\operatorname{ann}\left(N_{i} / N_{i-1}\right)$. If $L_{i} \neq 0$ then

$$
L_{i} \subset r-\operatorname{ann}\left(N_{i}+\alpha(F) / N_{i-1}+\alpha(F)\right)
$$

and so

$$
\operatorname{Bdim}_{R}\left(M / N_{i-1}+\alpha(F)\right)<\operatorname{Bdim}_{R}(M) .
$$

Therefore, we can assume that $L_{i}=0$. Since $M$ is torsion, $\operatorname{Ker}(\alpha) \neq 0$. Thus, since $F$ is projective, there exists $k \in \operatorname{Ker}(\alpha)$ and $\eta \in \operatorname{Hom}(F, R)$ with 
$\eta(k) \neq 0$. Hence $N_{i} R \eta(k) R \not \subset N_{i-1}$. Choose $x \in N_{i}$ such that $x \eta(k) \notin N_{i-1}$, and note that $x=\beta(g)$ for some $g \in F I$. Now define $\delta: F \rightarrow F I$ by $f \rightarrow g \eta(f)$. Then

$$
(\alpha+\beta \delta)(k)=\beta(g) \cdot \eta(k) \in\left(N_{i-1}+(\alpha+\beta \delta)(F)\right) \cap N_{i} .
$$

Since $N_{i} / N_{i-1}$ is critical this implies that

$\operatorname{Kdim}\left(N_{i}+(\alpha+\beta \delta)(F) / N_{i-1}+(\alpha+\beta \delta)(F)\right)<\operatorname{Kdim}\left(N_{i} / N_{i-1}\right) \leq \operatorname{Bdim}_{R}(M)$.

Of course $N_{i}+(\alpha+\beta \delta)(F)=N_{i}+\alpha(F)$, and so $\alpha+\beta \delta$ satisfies

$$
\operatorname{Bdim}_{R}\left(N / N_{i-1}+(\alpha+\beta \delta)(F)\right)<\operatorname{Bdim}_{R}(M)
$$

as required.

Corollary 4.4. Let $I$ be a nonzero two-sided ideal of $R$. Suppose that $M$ is torsion and that $\theta_{1}, \ldots, \theta_{s} \in \operatorname{Hom}(F, M)$ satisfy $\operatorname{Bdim}_{R}\left(M / \sum_{1}^{s} \theta_{i}(F)\right)<$ $\operatorname{Bdim}_{R}(M)$. Then there exist $\delta_{2}, \ldots, \delta_{s} \in \operatorname{Hom}(F, F I)$ such that $\theta=\theta_{1}+$ $\sum_{s}^{2} \theta_{i} \delta_{i}$ satisfies

$$
\operatorname{Bdim}_{R}(M / \theta(F))<\operatorname{Bdim}_{R}(M) .
$$

Proof. Consider the following inductive hypothesis:

$A(j)$ : Let $M$ be a finitely generated torsion right $R$-module. Let $\theta_{1}, \ldots, \theta_{j}$ $\in \operatorname{Hom}(F, M)$ with $\operatorname{Bdim}_{R}\left(M / \sum_{1}^{j} \theta_{i}(F)\right)<\operatorname{Bdim}_{R}(M)$. Then there exist $\delta_{2}, \ldots, \delta_{j} \in \operatorname{Hom}(F, F I)$ such that $\theta=\theta_{1}+\sum_{2}^{j} \theta_{i} \delta_{i}$ satisfies $\operatorname{Bdim}_{R}(M / \theta(F))$ $<\operatorname{Bdim}_{R}(M)$.

The case $j=2$ is given by Proposition 4.3. Suppose now that $A(j)$ is true for all $j \leq s-1$. We prove that $A(s)$ is also true. Assuming that the hypothesis of $A(s)$ holds, let $M^{\prime}=M / \theta_{1}(F)$. For $\phi: F \rightarrow M$ let $\bar{\phi}$ denote the induced map $F \rightarrow M^{\prime}$. Suppose now that $\operatorname{Bdim}_{R}\left(M^{\prime}\right)=\operatorname{Bdim}_{R}\left(M^{\prime} / \sum_{2}^{s} \bar{\theta}_{i}(F)\right)$. Then $\operatorname{Bdim}_{R}\left(M^{\prime}\right)=\operatorname{Bdim}_{R}\left(M^{\prime} / \sum_{2}^{s} \bar{\theta}_{i}(F)\right)<\operatorname{Bdim}_{R}(M)$, and so $\operatorname{Bdim}_{R}\left(M / \theta_{1}(F)\right)$ $<\operatorname{Bdim}_{R}(M)$. Therefore the result follows if we set $\delta_{2}=\cdots=\delta_{s}=0$.

Suppose that $\operatorname{Bdim}_{R}\left(M^{\prime} / \sum_{2}^{s} \bar{\theta}_{i}(F)\right)<\operatorname{Bdim}_{R}\left(M^{\prime}\right)$. By induction, there exist $\eta_{3}, \ldots, \eta_{s} \in \operatorname{Hom}(F, F I)$ such that if $\phi=\theta_{2}+\sum_{3}^{s} \theta_{i} \eta_{i}$, then

$$
\operatorname{Bdim}_{R}(M) \geq \operatorname{Bdim}_{R}\left(M^{\prime}\right)>\operatorname{Bdim}_{R}\left(M^{\prime} / \bar{\phi}(F)\right)=\operatorname{Bdim}_{R}\left(M / \theta_{1}(F)+\phi(F)\right) .
$$

Another application of the induction hypothesis yields a $\lambda \in \operatorname{Hom}(F, F I)$ such that $\operatorname{Bdim}_{R}\left(M /\left(\theta_{1}+\phi \lambda\right)(F)\right)<\operatorname{Bdim}_{R}(M)$. The result follows if we set $\delta_{2}=\lambda$ and $\delta_{i}=\eta_{i} \lambda$ for $3 \leq i \leq s$, completing the proof of $A(s)$.

\section{THE MAIN THEOREM}

Throughout this section let $M$ be a finitely generated right module over a right noetherian ring $R$ and let $F$ be a finitely generated projective right $R$ module. We begin by rephrasing Corollary 4.2 in terms of $g^{F}(M, P)$. 
Proposition 5.1. Let $P$ be a prime ideal of $R$ such that $0<g^{F}(M, P)<\infty$. Let $I$ be a two-sided ideal of $R$ such that $I \not \subset P$. If $M=\sum_{1}^{s} \theta_{i}(F)$ for $\theta_{i} \in \operatorname{Hom}(F, M)$ then there exist $\delta_{i} \in \operatorname{Hom}(F, F I)$ such that

$$
g^{F}\left(M /\left(\theta_{1}+\sum_{2}^{s} \theta_{i} \delta_{i}\right)(F), P\right)=g^{F}(M, P)-1 .
$$

We now combine Propositions 5.1 and 4.4 to show that $b^{F}(M, P)$ can be reduced simultaneously at a finite number of prime ideals.

Lemma 5.2. Let $P_{1}, \ldots, P_{m}$ be prime ideals of $R$ with $0<b^{F}\left(M, P_{i}\right)<\infty$ for $1 \leq i \leq m$. Suppose that $M=\sum_{1}^{s} \theta_{i}(F)$ for $\theta_{i} \in \operatorname{Hom}(F, M)$. Then there exist $\delta_{2}, \ldots, \delta_{s} \in \operatorname{End}(F)$ such that if $\theta=\theta_{1}+\sum_{2}^{s} \theta_{i} \delta_{i}$ then

$$
b^{F}\left(M / \theta(F), P_{i}\right) \leq b^{F}\left(M, P_{i}\right)-1
$$

for $1 \leq i \leq m$.

Proof. The proof is by induction on $m$. Note that $m=0$ is trivial. Suppose that $P_{1}, \ldots, P_{m}$ are ordered so that if $P_{i} \subsetneq P_{j}$ then $i>j$. By induction we can assume that $\delta_{2}, \ldots, \delta_{s}$ have been chosen such that $\phi=\theta_{1}+\sum_{2}^{s} \theta_{i} \delta_{i}$ satisfies

$$
b^{F}\left(M / \phi(F), P_{i}\right) \leq b^{F}\left(M, P_{i}\right)-1
$$

for $1 \leq i \leq m-1$. Note that $M=\phi(F)+\sum_{2}^{s} \theta_{i}(F)$.

Let $I=P_{1} \cap \cdots \cap P_{m-1}$, then $I \not \equiv 0\left(\bmod P_{m}\right)$. We now consider two cases. First suppose that $g^{F}\left(M, P_{m}\right) \neq 0$, then by Proposition 5.1 choose $\delta_{2}^{\prime}, \ldots, \delta_{s}^{\prime} \in \operatorname{Hom}(F, F I)$ such that $\theta=\phi+\sum_{2}^{s} \theta_{i} \delta_{i}^{\prime}$ satisfies

$$
g^{F}\left(M / \theta(F), P_{m}\right) \leq g^{F}\left(M, P_{m}\right)-1 .
$$

On the other hand, if $g^{F}\left(M, P_{m}\right)=0$ then choose $\delta_{2}^{\prime}, \ldots, \delta_{s}^{\prime} \in \operatorname{Hom}(F, F I)$ by Corollary 4.4 such that $\theta=\phi+\sum_{2}^{s} \theta_{i} \delta_{i}^{\prime}$ satisfies $\operatorname{Bdim}\left(M / \theta(F), P_{m}\right) \leq$ $\operatorname{Bdim}\left(M, P_{m}\right)-1$. In either case $b^{F}\left(M / \theta(F), P_{m}\right) \leq b^{F}\left(M, P_{m}\right)-1$.

Finally since $\theta(F) \equiv \phi(F)\left(\bmod P_{i}\right)$ for $1 \leq i \leq m-1$, then

$$
b^{F}\left(M / \theta(F), P_{i}\right) \leq b^{F}\left(M, P_{i}\right)-1
$$

fer $1 \leq i \leq m$.

The next lemma shows that to prove the theorem we will have to deal with only a finite number of prime ideals.

Lemma 5.3. Let $Y=J-\operatorname{Spec}(R)$. Suppose that $r-\operatorname{Kdim}(R)<\infty$ and that $F$ covers $M$. If $M \neq 0$ then there are only finitely many $J$-prime ideals $P$ for which $b^{F}(M, P)=b^{F}(M, Y)$.

Proof. If the result is false then there exists an integer $t \geq 0$ such that $b^{F}(M, P)$ $\geq \max \left\{1, b^{F}\left(M, Y_{t+1}\right)\right\}$ for infinitely many $P \in Y_{t}-Y_{t+1}$. Among all such 
modules choose one, say $M$, for which $t$ is a large as possible and, modulo this, such that $b^{F}\left(M, Y_{t+1}\right)$ is as small as possible.

If $b^{F}\left(M, Y_{t+1}\right)=0$ then $\operatorname{Bdim}\left(M, Y_{t+1}\right)=-1$. By Corollary 2.6 there are only finitely many primes $P \in Y_{t}-Y_{t+1}$ such that $\operatorname{Bdim}(M, P) \geq 0$, contradicting the hypothesis. So we can assume that $b^{F}\left(M, Y_{t+1}\right)>0$.

Let $P \in Y_{t}-Y_{t+1}$. Notice that if $g^{F}(M, P) \leq 1$ then

$$
b^{F}(M, P)=\operatorname{Bdim}(M, P)+1,
$$

and if $P$ also satisfies $b^{F}(M, P) \geq b^{F}\left(M, Y_{t+1}\right)$, then

$$
\operatorname{Bdim}(M, P) \geq \operatorname{Bdim}\left(M, Y_{t+1}\right) .
$$

Thus by Corollary 2.6 there are only finitely many $P \in Y_{t}-Y_{t+1}$ with $g^{F}(M, P)$ $\leq 1$ and $b^{F}(M, P) \geq b^{F}\left(M, Y_{t+1}\right)$. In particular the set $X=\left\{P \in Y_{t}-\right.$ $Y_{t+1}: g^{F}(M, P) \geq 2$ and $\left.b^{F}(M, P) \geq b^{F}\left(M, Y_{t+1}\right)\right\}$ is infinite.

Our choice of $t$ implies that $b^{F}(M, Q)=b^{F}\left(M, Y_{t+1}\right)$ for only finitely many $Q \in Y_{t+1}$. Since $F$ covers $M, g^{F}(M)<\infty$ by Proposition 3.4. Thus by Lemma 5.2 there exists $\theta \in \operatorname{Hom}(F, M)$ such that $b^{F}\left(M / \theta(F), Y_{t+1}\right) \leq$ $b^{F}\left(M, Y_{t+1}\right)-1$. On the other hand if $P \in X$ then

$$
g^{F}(M / \theta(F), P) \geq g^{F}(M, P)-1 \geq 1 .
$$

Hence $b^{F}(M / \theta(F), P) \geq b^{F}\left(M / \theta(F), Y_{t+1}\right)$ for $P \in X$, which contradicts either the maximality of $t$ or the minimality of $b^{F}\left(M, Y_{t+1}\right)$.

We can now prove the following generalisation of the Forster-Swan Theorem.

Theorem 5.4. Let $M$ be a finitely generated right module over a right noetherian ring $R$ with finite right Krull dimension. Let $F$ be a finitely generated projective right $R$-module that covers $M$. Then

$$
s^{F}(M) \leq b^{F}(M, J-\operatorname{Spec}(R)) .
$$

Proof. Let $Y=J-\operatorname{Spec}(R)$. We proceed by induction on $b^{F}(M, Y)$. If $b^{F}(M, Y)=0$ then $M=0$ by Proposition 2.2. By Proposition 3.4 we have that $M=\sum_{1}^{s} \theta_{i}(F)$ for $\theta_{i} \in \operatorname{Hom}(F, M)$. Suppose that $s>b^{F}(M, Y)>0$. By Lemma 5.3 there are only finitely many $J$-primes $P$ for which $b^{F}(M, P)=$ $b^{F}(M, Y)$. Thus by Lemma 5.2 there exist $\delta_{i} \in \operatorname{End}(F)$ such that if $\theta=$ $\theta_{1}+\sum_{2}^{s} \theta_{i} \delta_{i}$ and $\bar{M}=M / \theta(F)$ then $b^{F}(\bar{M}, Y) \leq b^{F}(M, Y)-1$. Note that $\bar{M}=\sum_{2}^{s} \bar{\theta}_{i}(F)$, where the bar denotes the canonical homomorphism $M \rightarrow \bar{M}$. So $s-1<b^{F}(\bar{M}, Y)$. Thus by induction there exist $\eta_{i} \in \operatorname{End}(F)$ such that $\bar{M}=\sum_{2}^{s-1}\left(\bar{\theta}_{i}+\bar{\theta}_{s} \bar{\eta}_{i}\right)(F)$. Hence

$$
M=\sum_{1}^{s-1}\left(\theta_{i}+\theta_{s} \eta_{i}\right)(F),
$$

where $\eta_{1}=\delta_{s}-\sum_{2}^{s-1} \eta_{i} \delta_{i}$. 
Corollary 5.5. Let $R, M, F$ be as in the theorem. Then

(a) $s(M) \leq b(M, J-\operatorname{Spec}(R))$.

(b) $s(M) \leq \sup \{g(M, P)+\operatorname{Kdim}(R / P): P \in J-\operatorname{Spec}(R)\}$.

Proof. (a) is the theorem with $F=R$. Since

$$
b(M, P) \leq g(M, P)+\mathrm{K} \operatorname{dim}(R / P)
$$

for every $P \in J-\operatorname{Spec}(R),(\mathrm{b})$ is an immediate consequence of $(\mathrm{a})$.

Another immediate consequence of Theorem 5.4 is the following result of Warfield [14].

Corollary 5.6. Let $R$ be an FBN ring with finite right Krull dimension, then

$$
s(M) \leq \sup \{\widehat{b}(M, P): P \in J-\operatorname{Spec}(R)\}
$$

where

$$
\begin{aligned}
\widehat{b}(M, P) & =g(M, P)+\mathrm{K} \operatorname{dim}(R / P) & & \text { if } g(M, P) \neq 0, \\
& =0 & & \text { otherwise. }
\end{aligned}
$$

Since a commutative noetherian ring is FBN, Corollary 5.6 enables us to recover the original result of Eisenbud and Evans [4].

We will end with an illustrative example drawn from [11]. Recall that the first Weyl algebra over $\mathbb{Z}$, denoted by $A_{1}(\mathbb{Z})$, is the $\mathbb{Z}$-algebra generated by $x$ and $y$ under the relation $x y-y x=1$.

Example 5.7. Let $R=A_{1}(\mathbb{Z}), M=R / x R$ and $X=\operatorname{Spec}(R)$. Then:

(a) $\sup \{g(M, P)+\operatorname{Kdim}(R / P): P \in X$ and $g(M, P) \neq 0\}=2$.

(b) There are infinitely many $P \in X$ with $g(M, P)+\operatorname{Kdim}(R / P)=2$.

(c) $b(M, \operatorname{Spec}(R))=b(M, 0)=\operatorname{Bdim}(M, 0)+1=3$.

This example shows that, if our definition of $b^{F}(M, P)$ were replaced by that of Stafford [11], as described at the end of $\S 3$, then Lemma 5.3 would fail.

Proof. We first recall a few elementary facts about the prime ideals of $R$, see [9] for details. The height one primes of $R$ are all of the form $p R$, for a prime $p \neq 0$ of $\mathbb{Z}$. Also $p R+x^{p} R$ and $p R+x^{p} R+y^{p} R$ are primes. Therefore we have a chain

$$
0 \subset p R \subset p R+x^{p} R \subset p R+x^{p} R+y^{p} R .
$$

Since $\operatorname{Kdim}(R)=3$, the factors of $R$ by the prime ideals of this chain must have Krull dimensions 3, 2, 1 and 0, respectively.

If $P=0$ or if $P$ is a prime ideal of height one, then $M / M P$ is torsion over $R / P$ and so $g(M, P)=0$. For any other prime ideal, if $g(M, P) \neq 0$ then $g(M, P)+\operatorname{Kdim}(R / P) \leq 2$. However if $P=p R+x^{p} R$ for some prime $p$, then

$$
M / M P=R / x R+p R \simeq x^{i} R+p R / x^{i+1} R+p R \simeq x^{p-1} R+p R / P
$$

for $1 \leq i \leq p-1$. We also have that $x^{p-1} R+p R / P$ is a uniform right ideal of $R / P$. Hence $\rho(M, P)=1, \rho(R, P)=p$ and $b(M, P)=g(M, P)+$ $\operatorname{Kdim}(R / P)=2$. This proves (a) and (b). 
To prove (c) it is enough to show that $\operatorname{Bdim}(M, 0)=2$. Notice that $x A_{1}(\mathbb{Q})$ is a maximal right ideal of $A_{1}(\mathbb{Q})$. Thus any proper factor $N$ of $R / x R$ is annihilated by some nonzero integer. It then follows from the previous paragraph that $\operatorname{Kdim}(N) \leq 1$. However since $R / x R$ is faithful and $\operatorname{Kdim}(R / x R)=2$, any submodule of $R / x R$ is faithful and has Krull dimension 2. Thus $\operatorname{Bdim}(M, 0)=2$, as required.

Acknowledgments. The material of this paper is part of my $\mathrm{PhD}$ thesis at the University of Leeds. I wish to thank my supervisor J. T. Stafford for suggesting the problem and for his help and encouragement, and J. C. McConnell and J. C. Robson for many enlightening conversations and comments. During the preparation of the thesis I received financial support from CNPq (Brazil) and an ORS Award (U.K.).

\section{REFERENCES}

1. S. C. Coutinho, Generating modules efficiently over non-commutative noetherian rings, $\mathrm{PhD}$ thesis, Leeds University, 1986.

2. __ Basic element theory of noncommutative noetherian rings, J. Algebra (to appear).

3. C. Dean and J. T. Stafford, A nonembeddable noetherian ring, J. Algebra 115 (1988), $175-181$.

4. D. Eisenbud and E. G. Evans, Jr., Generating modules efficiently: theorems from algebraic $K$-theory, J. Algebra 27 (1973), 278-305.

5. R. Gordon and J. C. Robson, Krull dimension, Mem. Amer. Math. Soc., No. 133, 1973.

6. N. Jacobson, Basic algebra II, Freeman, San Francisco, Calif., 1980.

7. A. V. Jategaonkar, Jacobson's conjecture and modules over fully bounded noetherian rings, J. Algebra 30 (1974), 103-121.

8. E. Kunz, Introduction to commutative algebra and algebraic geometry, Birkhäuser, Boston, Mass., 1985.

9. J. C. McConnell and J. C. Robson, Noncommutative noetherian rings, Wiley, London, 1987.

10. L. Rowen, Ring theory, Vol. I, Academic Press, San Diego, Calif., 1988.

11. J. T. Stafford, Generating modules efficiently: algebraic $K$-theory for noncommutative noetherian rings, J. Algebra 69 (1981), 312-346.

12. __ Generating modules efficiently over noncommutative rings, Lecture Notes in Math., vol. 924, Springer, New York, 1982, pp. 72-88.

13. __ Nonholonomic modules over Weyl algebras and enveloping algebras, Invent. Math. 79 (1985), 619-638.

14. R. B. Warfield, Jr., The number of generators of a module over a fully bounded ring, J. Algebra 66 (1980), 425-447.

Departamento de Matemática Aplicada, Instituto de Matemática UfRJ, C. Postal 68530, 21944 Rio DE JANEIRo, RJ, BRAZIL 